Article

\title{
SOCS3-Mediated Blockade Reveals Major Contribution of JAK2/STAT5 Signaling Pathway to Lactation and Proliferation of Dairy Cow Mammary Epithelial Cells in Vitro
}

\author{
Yu-Ling Huang, Feng Zhao, Chao-Chao Luo, Xia Zhang, Yu Si, Zhe Sun, Li Zhang, \\ Qing-Zhang Li and Xue-Jun Gao *
}

Key laboratory of Dairy Science of Education Ministry, Northeast Agricultural University, Harbin 150030, Heilongjiang, China; E-Mails: hyllw335474663@163.com. (Y.-L.H.); erjinzhi@126.com(F.Z.); luochaochao839505@163.com (C.-C.L.); zx291765746@126.com (X.Z.); siyu19880329@163.com(Y.S.); sunzhe1998@163.com (Z.S.); zhangli19782000@sohu.com (L.Z.); qingzhangli2004@163.com (Q.-Z.L.)

* Author to whom correspondence should be addressed; E-Mail: gaoxj5390@sina.com; Tel./Fax: +86-451-5519-0244.

Received: 12 August 2013; in revised form: 21 September 2013 / Accepted: 30 September 2013 / Published: 17 October 2013

\begin{abstract}
Suppressor of cytokine signaling 3 (SOCS3) is a cytokine-induced negative feedback-loop regulator of cytokine signaling. More and more evidence has proved it to be an inhibitor of signal transducers and activators of transcription 5 (STAT5). Here, we used dairy cow mammary epithelial cells (DCMECs) to analyze the function of SOCS3 and the interaction between SOCS3 and STAT5a. The expression of SOCS3 was found in cytoplasm and nucleus of DCMECs by fluorescent immunostaining. Overexpression and inhibition of SOCS3 brought a remarkable milk protein synthesis change through the regulation of JAK2/STAT5a pathway activity, and SOCS3 expression also decreased SREBP-1c expression and fatty acid synthesis. Inhibited STAT5a activation correlated with reduced SOCS3 expression, which indicated that SOCS3 gene might be one of the targets of STAT5a activation, DCMECs treated with L-methionine (Met) resulted in a decrease of SOCS3 expression. SOCS3 could also decrease cell proliferation and viability by CASY-TT detection. Together, our findings indicate that SOCS3 acts as an inhibitor of JAK2/STAT5a pathway and disturbs fatty acid synthesis by decreasing SREBP-1c expression, which validates its involvement in both milk protein synthesis and fat synthesis. In aggregate, these results reveal that low SOCS3 expression is required for milk synthesis and proliferation of DCMECs in vitro.
\end{abstract}


Keywords: SOCS3; dairy cow mammary epithelial cells; milk protein synthesis; milk fat synthesis; lactation

\section{Introduction}

Suppressor of cytokine signaling (SOCS) proteins are inhibitors of JAK/STAT pathway and act as a classical negative feedback loop that modulates cellular responsiveness to cytokines, first discovered in 1997 by different groups [1-3]. As a member of SOCS family, numerous biological functions have been attributed to SOCS3 activity, ranging from growth and development to lactation and apoptosis, from insulin resistance to obesity, as well as immunity and inflammatory response [4-6]. SOCS3 expression is stimulated by various cytokines, including growth hormone (GH), prolactin (PRL), insulin (INS), insulin-like growth factor-1 (IGF-1) and inhibited by glucocorticoids [7,8]. The interaction between SOCS3 and STAT5 has been intensively studied recently. SOCS3 has a potent inhibitory effect on STAT5, and its expression is STAT5 dependent in breast cancer cells [9], and appears to be independent from STAT5 activation in vivo [10] and not a prominent negative regulator of STAT5 signaling in mouse during pregnancy and lactation [11]. However, in other experiments SOCS3 was demonstrated to inhibit PRL induction of milk protein gene expression and STAT5 activation in vitro [12]. Whether SOCS3 is a negative feedback-loop regulator of the JAK2/STAT5 signaling pathway in dairy cow mammary epithelial cells (DCMECs) is not fully known, further experiments are needed to clarify this point. STAT5 was originally identified as a transcription factor that regulated the $\beta$-casein gene in response to PRL. It is encoded by two closely related genes, STAT5a and STAT5b [13]. STAT5 is a major factor controlling expression of milk protein genes and cell proliferation, which has largely been investigated in mouse and bovine [14-17]. These reports differ from that of Bionaz and Loor et al. [18], who supported a minor role of JAK2/STAT5 signaling for milk protein synthesis in bovine mammary gland in vivo. The function of STAT5 gene and regulatory networks linking STAT5 in milk production of DCMECs remains controversial. Whether SOCS3 participates in JAK2/STAT5 pathway to regulate milk protein synthesis in DCMECs is unknown.

The mammalian target of rapamycin (mTOR) in promoting protein synthesis has been well described, and sterol regulatory element binding protein (SREBP) plays an important role in regulating lipid synthesis [19]. Whether SOCS3 affects lactation through these pathways in DCMECs and the molecular mechanisms mediate their effects remain largely unknown. Studies have investigated the relationship between SOCS3 and SREBP-1c, but no study has been performed in DCMECs [20]. SOCS3 might be involved in regulation of milk fat synthesis in DCMECs [21]. Understanding the regulatory effects of individual amino acids (AAs) on milk protein synthesis is important for lactation. Methionine (Met) is an essential AA that plays fundamental roles in protein synthesis and a number of cellular processes including cell proliferation [22,23]. In low-amino-acid medium, expression of SOCS3 was increased in Huh-7 cells [24]. While no study has directly shown regulation of SOCS3 expression by Met, the effects of Met on the expression of SOCS3 remain largely elusive. The present study aims to establish direct actions of SOCS3 on lactation and proliferation of DCMECs, and to further elucidate the role of SOCS3-mediated blockade of JAK2/STAT5 signaling pathway. 


\section{Results and Discussion}

\subsection{SOCS3 Overexpression Decreased Cell Proliferation and Negatively Regulated JAK2/STAT5a} Signaling Pathway as well as Fatty Acid Synthesis in DCMECs

SOCS3 is an established negative regulator of STATs. To test whether SOCS3 was able to prevent STAT5a activation in DCMECs, SOCS3 was overexpressed and the expressions of related genes were detected by qRT-PCR and WB at $48 \mathrm{~h}$, respectively. Meanwhile cell proliferation and viability were examined by CASY. SOCS3 mRNA expression was significantly increased in pGCMV-IRES-EGFP-SOCS3 groups (Figure 1A). The SOCS3 protein expression in pGCMV-IRES-EGFP-SOCS3 groups was markedly higher than that in pGCMV-IRES-EGFP group (Figure 1B,C). We observed a significant $60 \%-65 \%$ increase of SOCS3 protein in DCMECs. It indicated that SOCS3 was stable-highly expressed in DCMECs infected with pGCMV-IRES-EGFP-SOCS3. The mRNA levels and protein expressions of STAT5a, p-STAT5a, mTOR, p-mTOR and $\beta$-casein were obviously reduced in cells transfected with SOCS3 compared with empty vector group (Figure 1A-C). Here we demonstrated that overexpression of SOCS3 in DCMECs significantly inhibited cell growth and viability (Figure 1D,E). In the present study, we found that SOCS3 protein was distributed both in the cytoplasm and nucleus in DCMECs. Infrequently, cells displayed a speckled nuclear SOCS3 expression pattern (Figure 1F). In experiments of overexpression of SOCS3 protein, we found that SOCS3 had higher levels of nuclear localization when their levels rose (Figure 1G,I). Additionally, localization of p-STAT5a was assessed by immunofluorescence after SOCS3 overexpression. p-STAT5a protein was distributed both in the cytoplasm and nucleus. After transfection with SOCS3 gene, p-STAT5a expression level was down-regulated in nucleus (Figure $1 \mathrm{H}, \mathrm{J}$ ), which was consistent with the result of WB.

To demonstrate the effect of SOCS3 on lipid synthesis, we detected expressions of key genes on lipid metabolism such as SREBP-1c, fatty acid synthase (FAS), acetyl-CoA carboxylase (ACC) and stearoyl-CoA desaturase (SCD) mRNA levels. Results showed that overexpression of SOCS3 downregulated SREBP-1c, FAS, ACC and SCD mRNA levels (Figure 1A). The expression of SREBP-1c protein by western blotting was at the same trend with mRNA level. SOCS3 evidently inhibited the expression of SREBP-1c protein (Figure 1B,C). These results revealed that overexpression of SOCS3 inhibited SREBP-1c expression and decreased fatty acid synthesis.

\subsection{Inhibition of SOCS3 Increased the Expressions of Key Genes on Lactation and Proliferation of DCMECs}

Using siRNA targeted to SOCS3, we examined the effect of SOCS3 silencing on lactation and proliferation in DCMECs. SOCS3 was inhibited and the expressions of certain genes were detected by qRT-PCR and WB at $24 \mathrm{~h}$, respectively. Meanwhile cell proliferation and viability were examined by CASY. SOCS3 knock-down after siRNA transfection was verified using qRT-PCR. SOCS3 inhibition increased the expression of STAT5a, p-STAT5a, mTOR, p-mTOR, SREBP-1c, FAS, ACC, SCD and $\beta$-casein in cells transfected with SOCS3 compared with negative control group (Figure 2A). The mRNA levels and protein expressions of different genes were shown in Figure 2A-C. Silencing of SOCS3 resulted in increased proliferation and viability (Figure 2D,E). Additionally, localization of 
p-STAT5a was assessed by immunofluorescence after SOCS3 inhibition. p-STAT5a protein expression was up-regulated in nucleus (Figure 2F,G), which was consistent with the result of WB.

Figure 1. Effect of SOCS3 overexpression on lactation and proliferation in DCMECs. DCMECs were divided into three groups: transfected with a SOCS3 expression construct or empty vector (control group), nontransfected, grew for $48 \mathrm{~h}$. (A) Gene expressions in DCMECs growing for $48 \mathrm{~h}$ were detected using qRT-PCR and normalized to $\beta$-actin mRNA expression; (B) Western blotting results of SOCS3, STAT5a, p-STAT5a, mTOR, p-mTOR, SREBP-1c, $\beta$-casein and $\beta$-actin in DCMECs growing for $48 \mathrm{~h}$ after SOCS3 overexpression; (C) Results of gray scale scan of SOCS3, STAT5a, p-STAT5a, mTOR, p-mTOR, SREBP-1c and $\beta$-casein/ $\beta$-actin relative fold by western blotting after SOCS3 overexpression; (D,E) Proliferation and viability of DCMECs growing for $24 \mathrm{~h}$ were measured using CASY; (F) Localization of SOCS3. SOCS3 (green), nuclei(red). Scale bars $=75 \mu \mathrm{m}$. (G) Overexpressed SOCS3 proteins had higher levels of nuclear localization of DCMECs. a: Cells were transfected with a SOCS3 expression construct and grew for $24 \mathrm{~h}$; b: Cells were transfected with an empty vector and grew for $24 \mathrm{~h}$; c: Nontransfected cells. SOCS3(green), Giantin(Red) and DAPI(blue). Scale bars $=20 \mu \mathrm{m}$; (H) Immunofluorescent staining for p-STAT5a was performed on DCMECs. a: Cells were transfected with a SOCS3 expression construct and grew for $24 \mathrm{~h}$; Scale bars $=75 \mu \mathrm{m}$; b: Cells were transfected with an empty vector and grew for $24 \mathrm{~h}$; Scale bars $=20 \mu \mathrm{m}$; c: Nontransfected cells. p-STAT5 (green), Giantian (red) and nuclei were counterstained with DAPI (blue). Scale bars $=20 \mu \mathrm{m}$; (I,J) The analysis of fluorescence staining after SOCS3 overexpression. Different superscripts denote significant difference relative to controls. $*$ or $* *$ means significant difference compared with negative control $(p<0.05$ or $p<0.01)$.

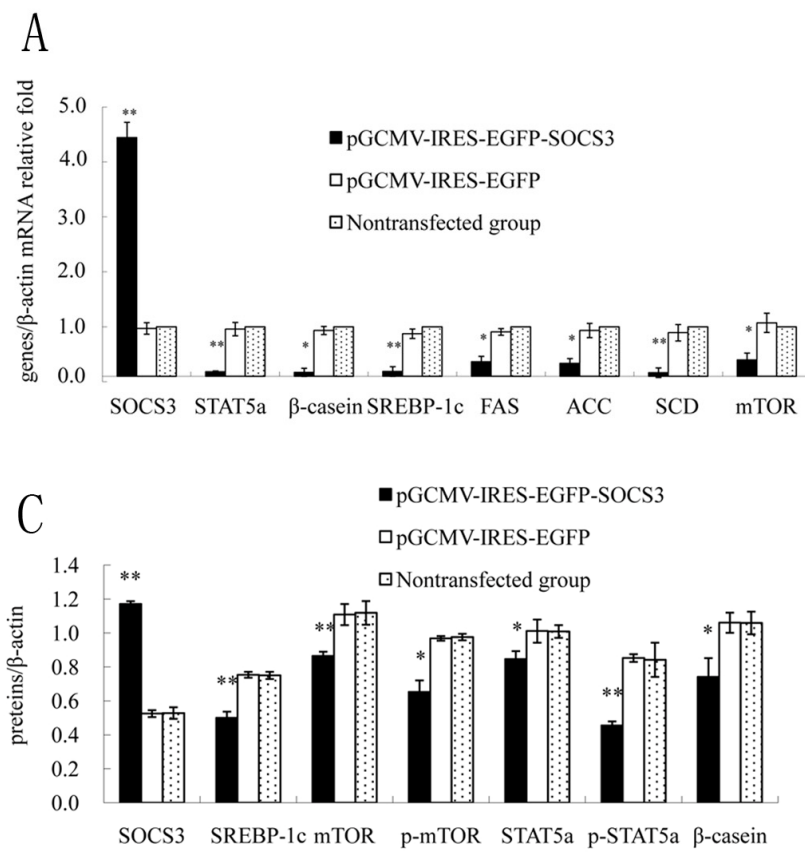

$\mathrm{B}$

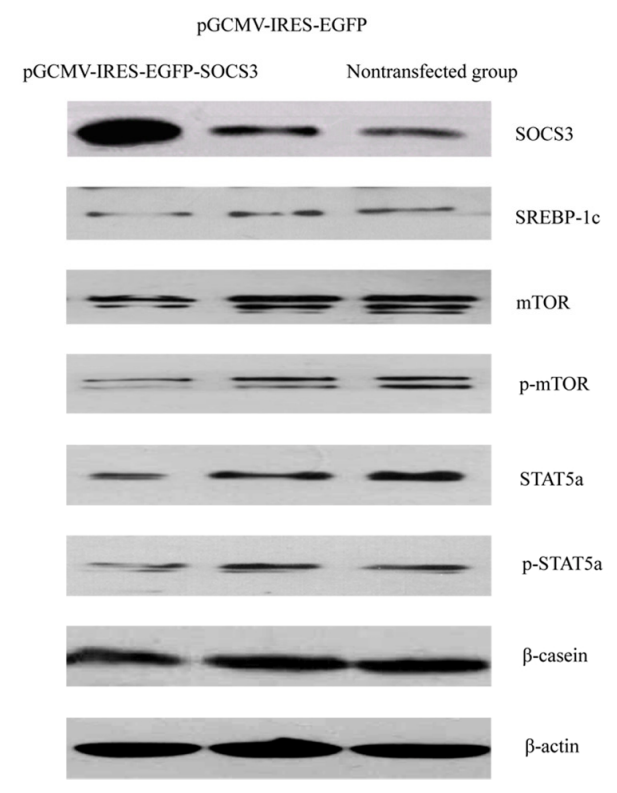


Figure 1. Cont.

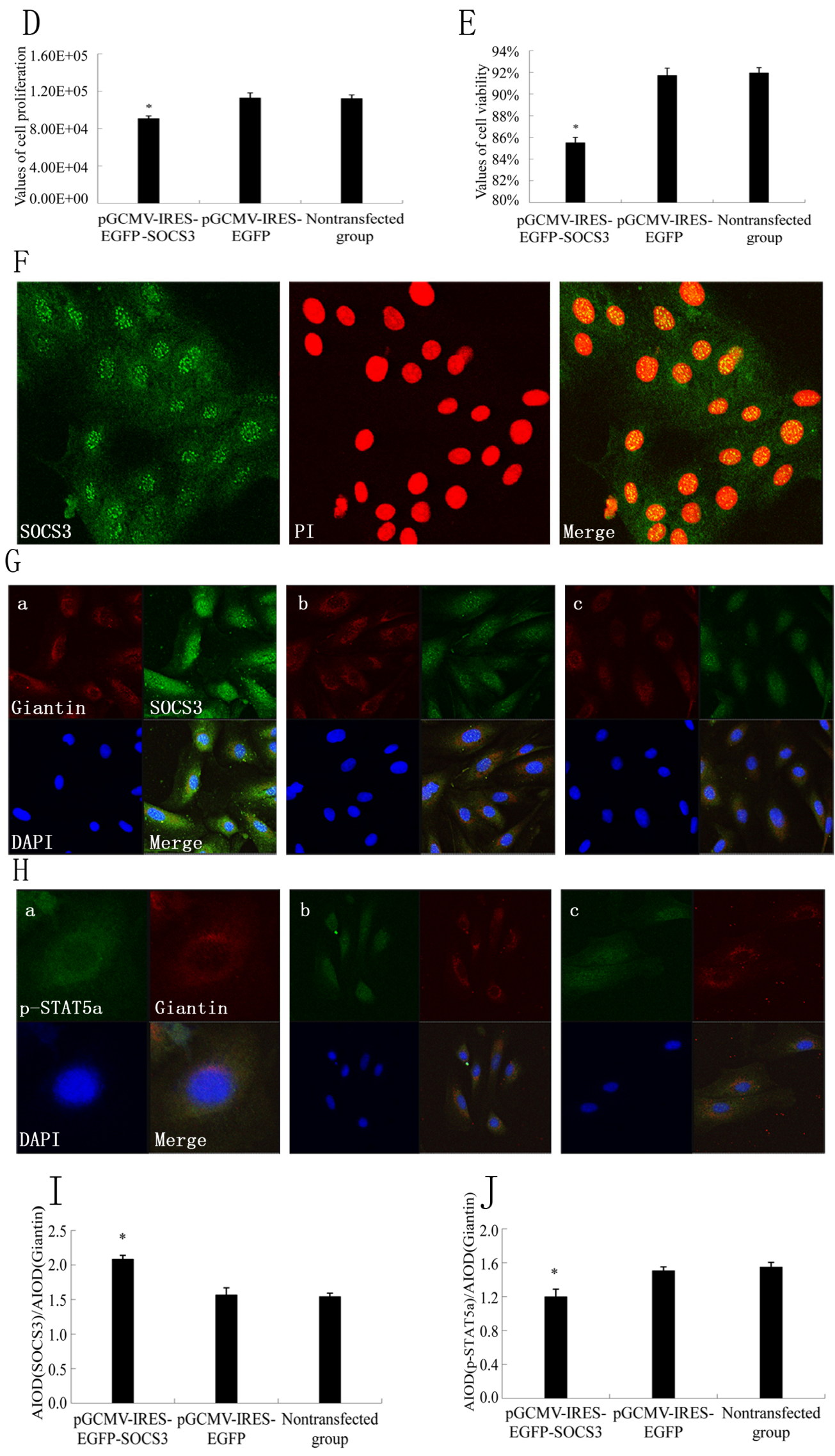


Figure 2. Effect of SOCS3 inhibition on lactation and proliferation of DCMECs. DCMECs were divided into three groups: transfected with a SOCS3 siRNA or negative control (control group), nontransfected, grew for $24 \mathrm{~h}$. (A) Relative mRNA expression of different genes in DCMECs growing for $24 \mathrm{~h}$ were measured by qRT-PCR after SOCS3 inhibition; (B) Western blotting results of SOCS3, STAT5a, p-STAT5a, mTOR, p-mTOR, SREBP-1c, $\beta$-casein and $\beta$-actin after SOCS3 inhibition in DCMECs growing for $24 \mathrm{~h}$; (C) Results of gray scale scan of SOCS3, STAT5a, p-STAT5a, mTOR, p-mTOR, SREBP-1c and $\beta$-casein $/ \beta$-actin relative fold by western blotting after SOCS3 inhibition at $24 \mathrm{~h}$; (D,E) Proliferation and viability of DCMECs growing for $24 \mathrm{~h}$ were measured using CASY; (F) Fluorescence staining after SOCS3 inhibition. a: Cells were transfected with SOCS3 siRNA; b: Negative control; c: Nontransfected cells. Scale bars $=75 \mu \mathrm{m}$ in a and b; $20 \mu \mathrm{m}$ in c; (G) The analysis of fluorescence staining after SOCS3 inhibition. * or ** means significant difference compared with negative control group $(p<0.05$ or $p<0.01$ ).

A

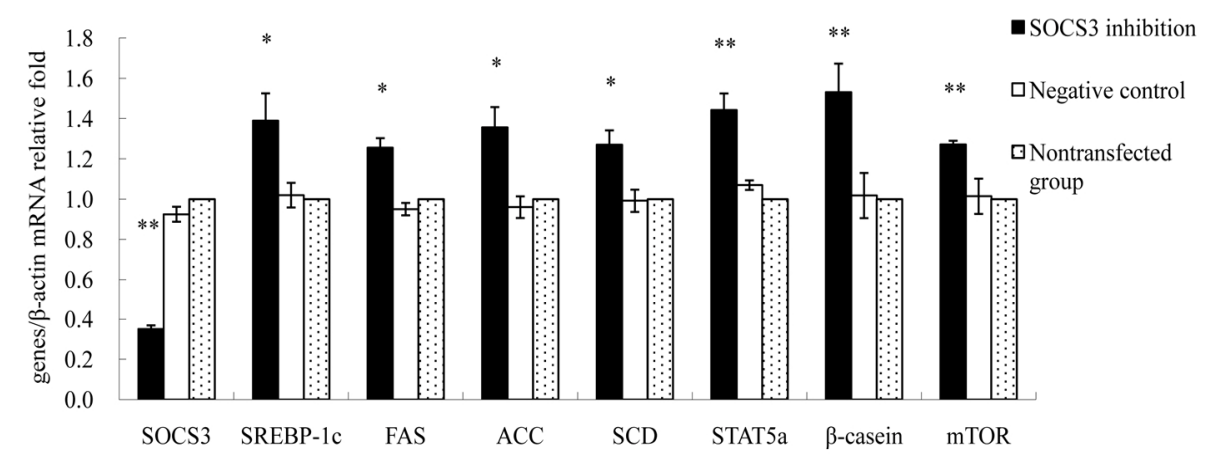

B

C

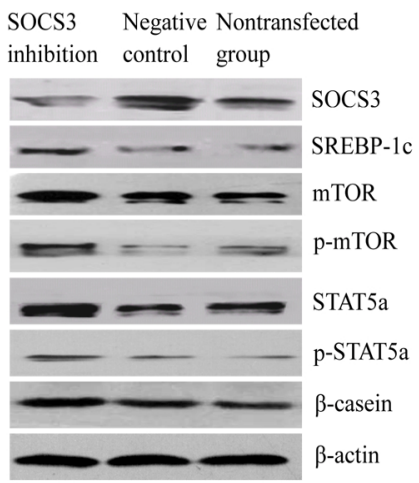

D

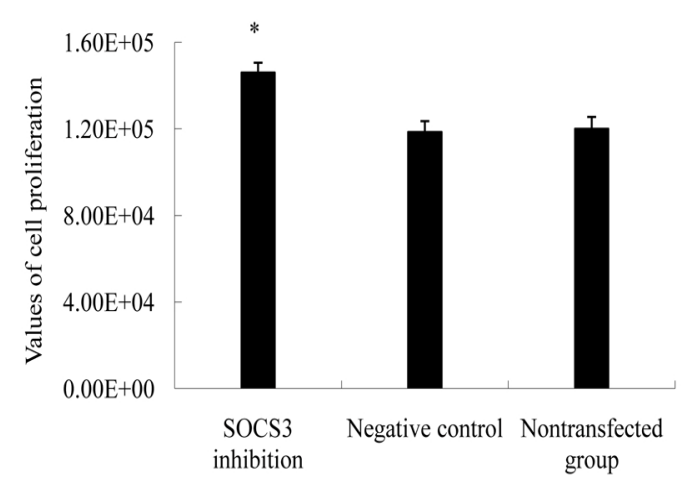

- SOCS3 inhibition 口Negative control $\square$ Nontransfected group

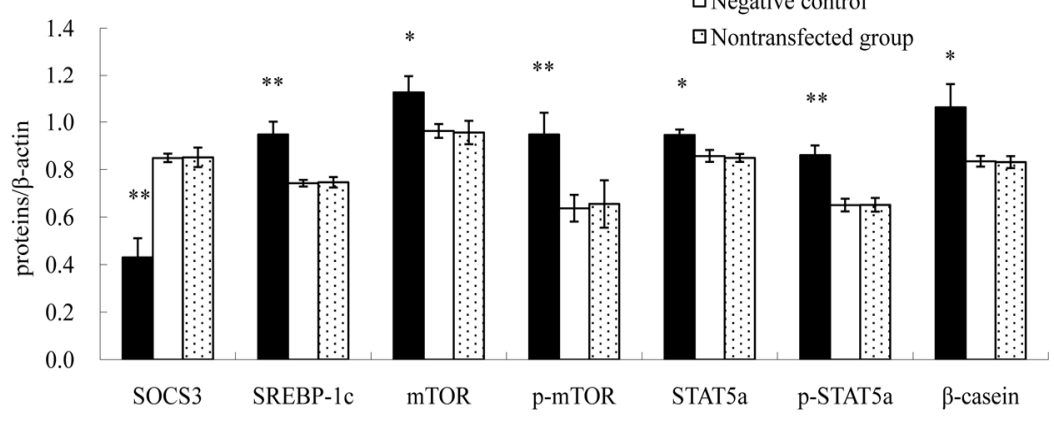

E

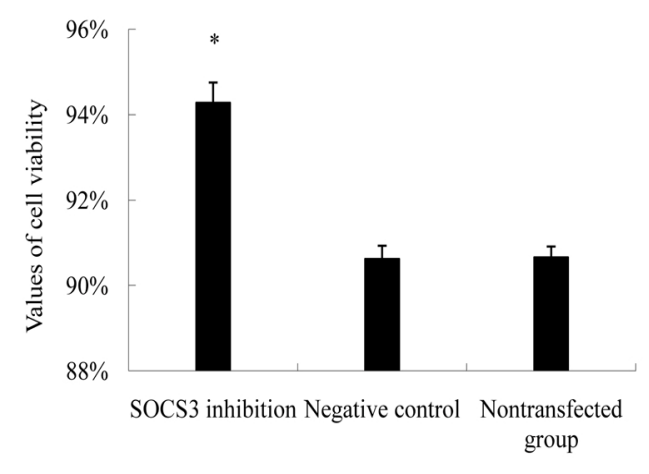


Figure 2. Cont.

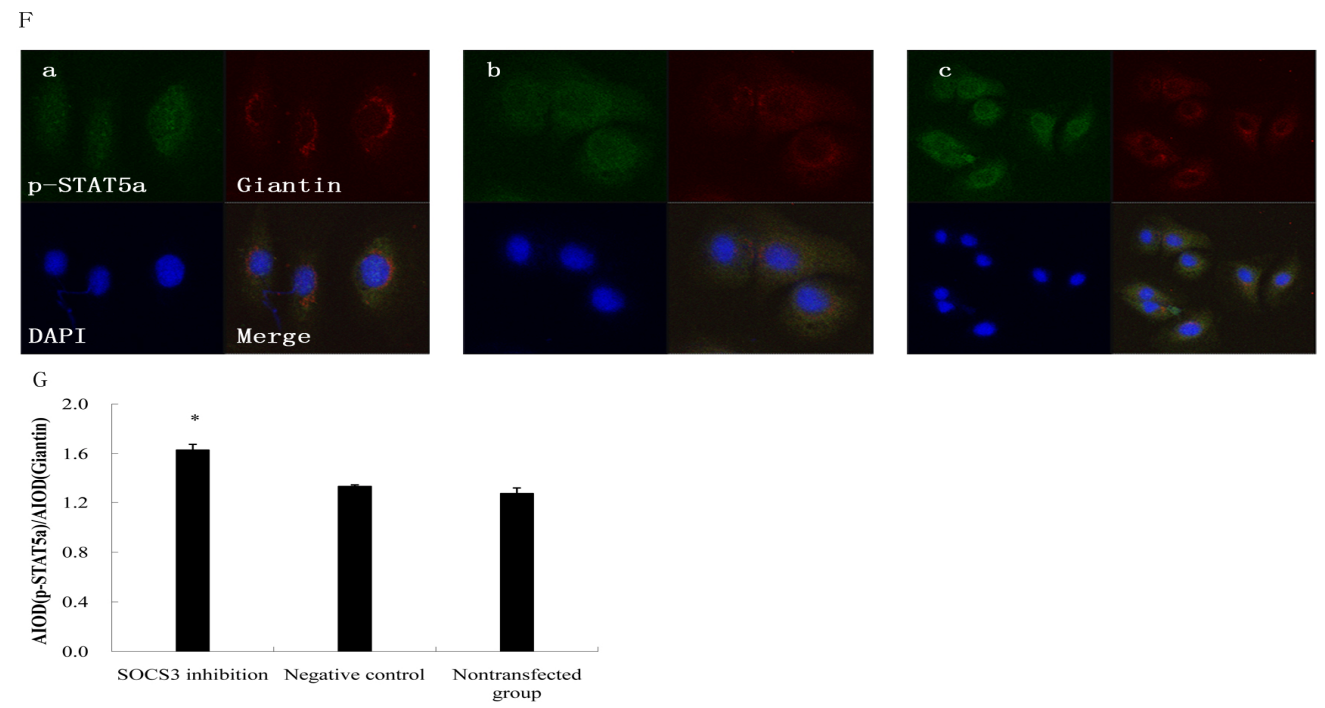

\subsection{STAT5a was Required for SOCS3 Activation in DCMECs}

It was investigated whether STAT5a played an important role in the mammary gland on the transcriptional regulation of SOCS3. Silencing of STAT5a gene expression was made by transfection with STAT5a siRNA for $24 \mathrm{~h}$. In STAT5a siRNA-treated cells, expression of p-STAT5a was low and difficult to detect (Figure 3A). We assessed the effect of silencing of STAT5a gene expression on SOCS3 in DCMECs, qRT-PCR and WB were used to investigate mRNA levels and protein expressions of STAT5a, p-STAT5a, SOCS3, $\beta$-casein, respectively. Data showed that they were all downregulated in STAT5a inhibition group compared with negative control group (Figure 3B-D), suggesting that this transcription factor was responsible for SOCS3 expression in DCMECs.

Figure 3. STAT5a was necessary for SOCS3 expression in DCMECs. DCMECs were divided into three groups: transfected with a STAT5a siRNA or negative control (control group), nontransfected, grew for 48 h. (A) Relative mRNA levels of STAT5a, SOCS3, $\beta$-casein genes in DCMECs growing for $24 \mathrm{~h}$ were measured by qRT-PCR after STAT5 inhibition. (B) Western blotting analysis of STAT5a, p-STAT5a, SOCS3, $\beta$-casein in DCMECs growing for $24 \mathrm{~h}$ subjected to STAT5a inhibition. $\beta$-actin served as loading control; (C) Results of gray scale scan of STAT5a, p-STAT5a, SOCS3 and $\beta$-casein/ $\beta$-actin relative fold by western blotting after STAT5a inhibition at $24 \mathrm{~h} . *$ or ** means significant difference compared with negative control $(p<0.05$ or $p<0.01)$.
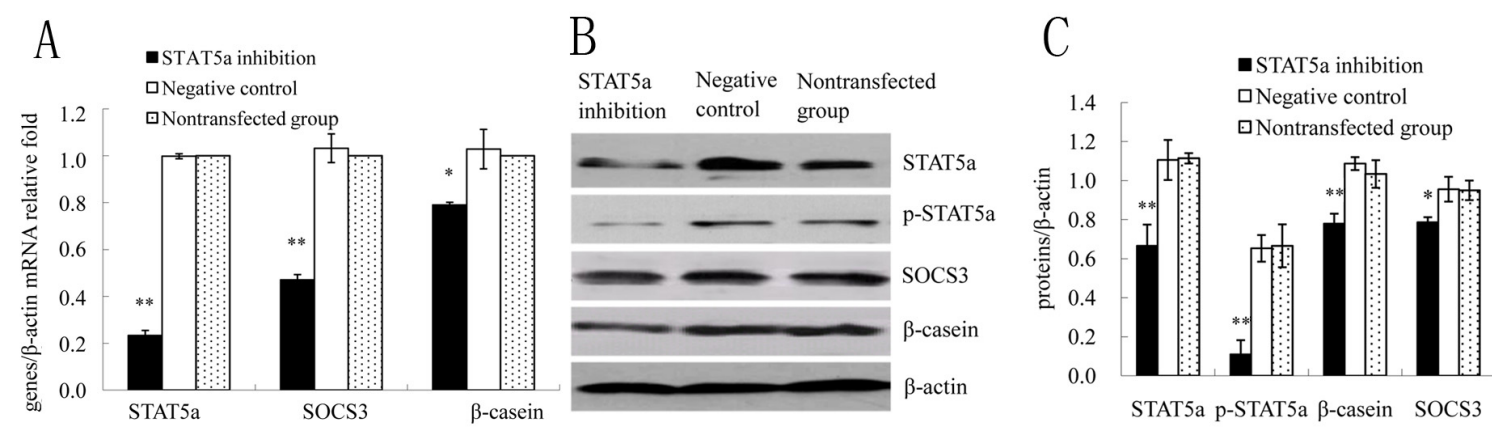


\subsection{The Effect of Met on SOCS3 to Regulate $\beta$-Casein Expression and Cell Proliferation}

To determine the effect of Met on $\beta$-casein expression and cell proliferation in DCMECs, the cells were treated with Met $\left(0.6 \mathrm{mmol} \mathrm{L}^{-1}\right)$ and harvested at $24 \mathrm{~h}$ after treatment, according to our laboratory's previous description [25]. Cell viability and proliferation were determined with CASY. The mRNA levels and protein expressions of SOCS3, STAT5a, p-STAT5a, mTOR, p-mTOR and $\beta$-casein were assessed by qRT-PCR and western blotting, respectively. Treatment with Met resulted in markedly increasing of mRNA levels of mTOR, STAT5a, $\beta$-casein (Figure 4A) as well as protein expressions of STAT5a and p-STAT5a, mTOR and p-mTOR, $\beta$-casein (Figure 4B,C), whereas the mRNA level and protein expression of SOCS3 were both decreased (Figure 4B,C). Results also showed that in comparison to the control group, cell viability and proliferation significantly were increased (Figure 4D,E). These data indicated that the expression of SOCS3 was inhibited by Met.

Figure 4. Effect of Met on SOCS3 to regulate $\beta$-casein expression and cell proliferation in DCMECs. DCMECs were treated with Met $\left(0.6 \mathrm{mmol} \mathrm{L}^{-1}\right)$ for $24 \mathrm{~h}$, not treated (control). (A) Changes of mRNA levels of key genes on milk protein synthesis were detected by qRT-PCR; (B) Western blotting results of SOCS3, STAT5a, p-STAT5a, mTOR, p-mTOR, $\beta$-casein and $\beta$-actin at $24 \mathrm{~h}$ after treatment with Met; $(\mathbf{C})$ Results of gray scale scan of SOCS3, STAT5a, p-STAT5a, mTOR, p-mTOR, $\beta$-casein/ $\beta$-actin relative fold by western blotting after treatment with Met; (D,E) Cell proliferation and viability were determined by the CASY assay. Different superscripts denote significant differences relative to controls. * or ** means significant difference compared with negative control $(p<0.05$ or $p<0.01)$.
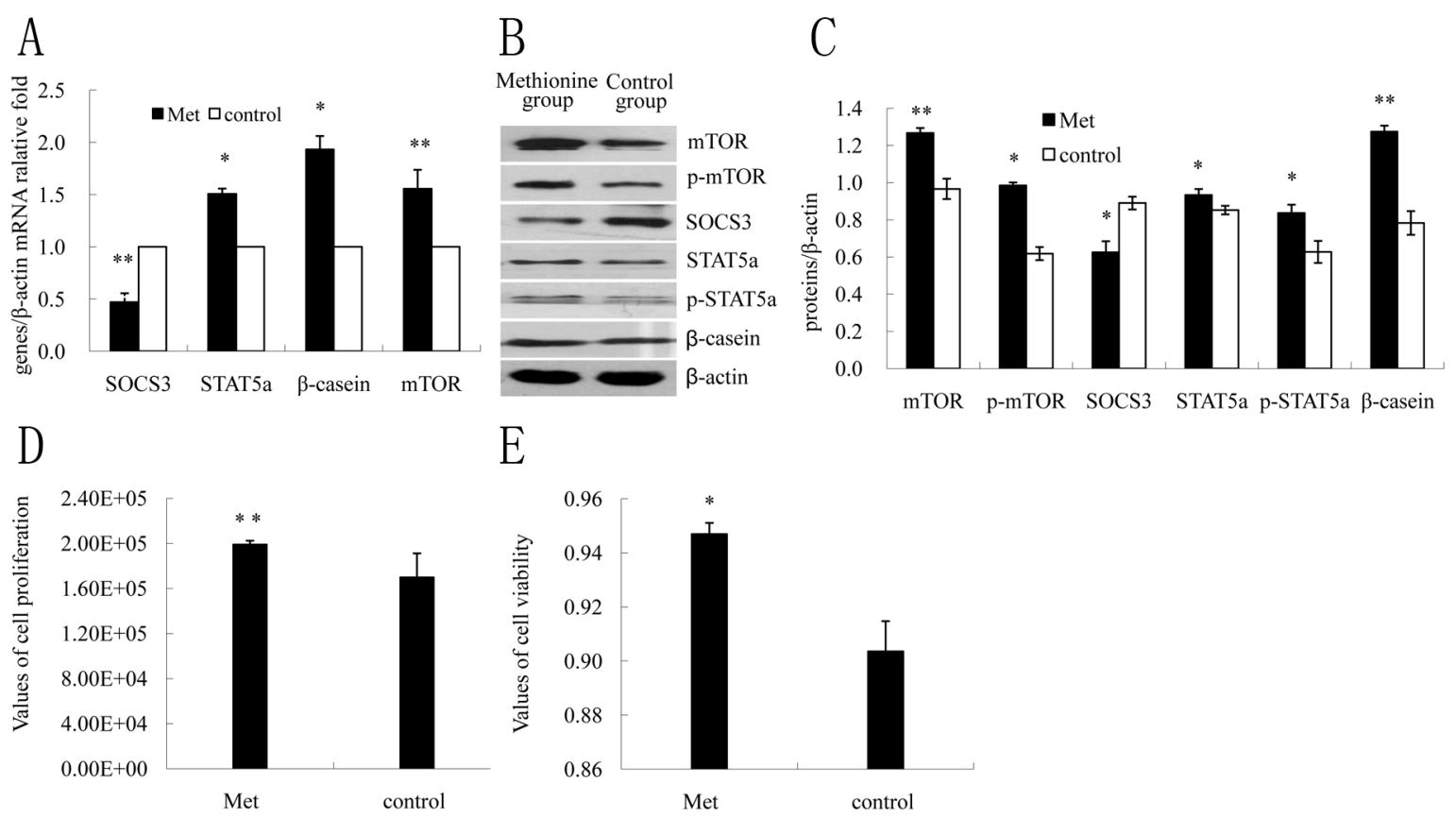

\subsection{Discussion}

By the experiments of overexpression and inhibition of SOCS3, we demonstrated that SOCS3 inhibited $\beta$-casein gene expression and cell proliferation as an inhibitor of JAK2/STAT5a signaling pathway in DCMECs. The relative level of STAT5a transcript and the protein expressions of STAT5a 
and p-STAT5a in transfected cells correlated with expression of $\beta$-casein in DCMECs. More recent studies in mice and human provide a direct link between the SOCS3 and STAT5 [26,27]. SOCS3 serves as a negative regulator of JAK-STAT signalling via several distinct mechanisms, but there is no study reporting the negative feedback mechanism through its direct interaction with the STAT5 protein, though the general trend of their results indicates a role of SOCS3 in attenuating STAT5 expression $[28,29]$. Together, these data indicate that SOCS3, presumably through the inhibition of STAT5a activation, is an inhibitor of protein synthesis in DCMECs.

The role of STAT5a/b in mammary gland has been controversially discussed, with conflicting evidence depending on the model studied. However, in mouse and bovine mammary gland, increasing evidence suggest that STAT5a is crucial for mammary development and milk protein expression [30,31]. Thus, we investigated whether STAT5a played the same role in DCMECs. The inhibition of STAT5a resulted in decrease of mRNA level and protein expression of $\beta$-casein. This result is consistent with previous studies that STAT5 deficiency in mice mammary gland leads to a near-complete loss of lobuloalveolar development, a reduction in expression of milk protein genes during pregnancy and lactation failure $[32,33]$. Based on these findings, it is concluded that SOCS3 participates in JAK2-STAT5a pathway to regulate milk protein synthesis in DCMECs.

To address the mechanism of interaction between STAT5a and SOCS3, we sought to determine if overexpressing and inhibiting SOCS3 proteins changed activated STAT5a protein levels in a manner that was dependent on SOCS3 localization, we used immunofluorescence to observe the localization of SOCS3, we found that SOCS3 protein was distributed both in the cytoplasm and nucleus and overexpressed SOCS3 protein primarily localized in the nucleus where they might reduce the activity of STAT5a protein in DCMECs. The level of p-STAT5a increased in the nucleus after inhibition of SOCS3. Our results suggest that the expression and subcellular localization of p-STAT5a are regulated by SOCS3, which would provide an evaluation at the cellular level by which SOCS3 inhibits STAT5a activity. Therefore, these results are consistent with the Kyeong-Hee Lee who found the translocation of SOCS3 from the cytoplasm to the nucleus under certain biological conditions [34], thus we predict that there is a possible mechanism that a protein such as STAT5a interacted with SOCS3 might bring it into the nucleus.

Here, SOCS3 expression in DCMECs was dependent on STAT5a, as evidenced by the lack of SOCS3 expression in DCMECs with siRNA targeted to STAT5a. Considering with other reports, we deduced that SOCS3 genes might be one of STAT5a activation targets. This result was consistent with the report of Ehrentraut $\mathrm{S}$ et al. that inhibition of STAT5 activation by treatment with the STAT5 inhibitor pimozide was accompanied by dose-dependent losses of SOCS2/3 expression in all three PCM1-JAK2 cell lines (human leukemia/lymphoma cell lines), MAC-1/2A/2B [35]. This result differed from that of Le Provost et al. [36], who found STAT5a was not involved in the stimulation of the expression of SOCS3 in virgin mice mamary gland tissue. To determine whether SOCS3 expression through STAT5a, it is essential to identify the STAT5-binding sites in the SOCS3 gene promoter. STAT5 could be recruited to the STAT-response element (pSRE) of the SOCS3 promoter to initiate transcription of it in human and sequence analysis of the mouse SOCS3 promoter region revealed STAT5 binding site [9,37]. According to these, we inferred that activated STAT5a might bind to the promoter of SOCS3 in DCMECs. To sum up, SOCS3 is a negative feedback-loop regulator of the JAK2/STAT5a signaling pathway in DCMECs. Briefly, STAT5a increases SOCS3 expression, and then SOCS3 in turn acts to limit further STAT5a signaling, thus it is realized that they complete an 
intracellular negative feedback loop. This is the first report that SOCS3 inhibits milk protein synthesis in DCMECs. Up to date, the function and mechanism of SOCS3 in milk protein synthesis is far more established.

Recent studies in rodents and ruminants have highlighted a role of mTOR in the regulation of milk protein synthesis [38,39]. In this study we also found SOCS3 inhibited the expression and phosphorylation of mTOR. This negative effect of SOCS3 does not appear to be DCMECs specific because it has been described recently also in murine models of oxygen-induced retinopathy and cancer [40]. This result further revealed that SOCS3 involved in regulation of milk synthesis in DCMECs. The precise mechanism by which SOCS3 regulates mTOR remains to be elucidated.

The number and activity of DCEMCs are determining factors for milk production [41]. Thus, we next elucidated the effects of SOCS3 on cell proliferation. By experiments with CASY, we found overexpression of SOCS3 inhibited cellular growth, whereas SOCS3 inhibition induced cellular growth. Our data suggest that SOCS3 itself acts as an inhibitor of cell proliferation. Furthermore, SOCS3 has a role as negative regulator of STAT5a or mTOR phosphorylation. These results suggest that SOCS3 might inhibit cellular proliferation at least via a STAT5a or mTOR independent mechanism. This finding is similar to studies carried out in human lung cancer where SOCS3 also yielded a decrease of cellular growth [42].

Here, our results verify that SOCS3 affects SREBP-1c expression and its downstream regulatory targets, FAS, SCD and ACC in DCMECs. This result contrasts markedly with earlier studies, in insulin signaling SOCS3 overexpression increased SREBP-1c and its downstream regulatory targets in livers of morbidly obese women and obese diabetic mice [43,44]. It is unclear whether the binding between SREBP-1c and SOCS3 is direct or through expressions of key genes on milk synthesis such as mTOR or STAT5a. SREBP-1 plays an important role in integrated regulation of lipid synthesis in bovine [45]. The discovery of a connection between SOCS3 and SREBP-1c opens a new chapter in our understanding of the molecular mechanisms regulating lipid synthesis.

Met is an essential amino acid that serves as a substrate for protein synthesis. Here, treatment with Met resulted in more cell proliferation and marked increase of expression of mTOR, STAT5a and $\beta$-casein. Surprisingly, the abundance of SOCS3 did not increase but actually decreased. The presence of essential AAs led to directly induced mTOR activation such as leucyl-tRNA synthetase (LRS) directly bound to Rag GTPase to activate mTORC1 [46], and contributed to enhancement of milk protein gene expression [47]. We inferred that the positive effect of Met on mTOR activation would be the main reason to decrease SOCS3 expression. But the mechanism is not known. Combined with our report, we predict that maintaining SOCS3 in a low level state is important to ensure lactation and proliferation of mammary epithelium instead of initiating apoptosis. Met can up-regulate milk protein synthesis partly via the inhibition of expression of SOCS3 expression.

\section{Experimental}

\subsection{Cell Preparation and Treatments}

DCMECs were cultured according to the previous method of our laboratory reported by Lu et al. [48]. The cells were cultured in Dulbecco's modified Eagle medium: Nutrient Mixture 12 
(DMEM: F12) supplemented with 10\% fetal bovine serum (FBS), insulin (bovine, $5 \mu \mathrm{g} \mathrm{mL}{ }^{-1}$ ), hydrocortisone $\left(1 \mu \mathrm{g} \mathrm{mL}^{-1}\right)$, penicillin $\left(100 \mathrm{U} \mathrm{mL}^{-1}\right)$ and streptomycin $\left(0.1 \mathrm{mg} \mathrm{mL}{ }^{-1}\right)$. Prior to the experimental treatments, cells were plated at $3 \times 10^{4}$ cells $\mathrm{cm}^{-2}$ in six-well plates, and the medium was replaced with DMEM: F12 containing insulin $\left(5 \mu \mathrm{g} \mathrm{mL} L^{-1}\right)$, hydrocortisone $\left(1 \mu \mathrm{g} \mathrm{mL}{ }^{-1}\right)$, and prolactin (ovine, $3 \mu \mathrm{g} \mathrm{mL}^{-1}$ ) without FBS.

\subsection{Cell Viability Assay}

Cell viability was determined with a CASY TT Analyser System (Schärfe System GmbH, Reutlingen, Germany) according to the manufacturer's instructions. After calibration with dead and vital DCMECs cells, cursor positions were set to 11.75 to $50.00 \mu \mathrm{m}$ (evaluation cursor) and 7.63 to $50.00 \mu \mathrm{m}$ (standardization cursor). Cells were trypsinized. The cells diluted with CASY electrolyte solution $(1: 100)$ was examined using CASY-TT, $100 \mu \mathrm{L}$ aliquots were analyzed in triplicate.

\subsection{RNA Extraction and Quantitative Real-Time PCR}

Total RNA extraction of DCMECs was performed using Trizol reagent (Invitrogen, Carlsbad, CA, USA). Subsequently, integrity and quality of RNA were quantified by gel analysis and reverse transcribed into cDNA using thermoscript reverse transcriptase (TaKaRa, Dalian, China) according to the manufacturer's protocol. Quantitative real-time PCR reactions were performed using real-time PCR Kit Sensimix TM SYBR\&Flurescen, and the analysis was performed by an ABI PRISM 7300 RT-PCR System (Applied Biosystems, Foster City, CA, USA) in a total volume of $20 \mu \mathrm{L}$ using 96-well microwell plates. All the following gene mRNA were normalized to $\beta$-actin mRNA level. The primers of these gene transcripts were as follows: SOCS3: sense5'-GAGAAGATCCCTCTGGTGTTGA-3', antisense5'-GGTCCAGGAACTCCCGAAT-3'; STAT5a: sense5'-GTCCCTTCCCGTGGTTGT-3', antisense5'-CGGCCTTGAATTTCATGTTG-3'; $\beta$-casein: sense5'-AACAGCCTCCCACAAAAC-3', antisense5'-AGCCATAGCCTCCTTCAC-3; SREBP-1c: sense5'-AGTAGCAGCGGTGGAAGT-3', antisense5'-GCAGCGGCTCTGGATT-3'; mTOR: sense5'-ATGCTGTCCCTGGTCCTTATG-3', antisense5'-GGGTCAGAGAGTGGCCTTCAA-3'; FAS: sense5'-CCACGGCTGTCGGTAAT-3', antisense5'-CGCTCCCACTCATCCTG-3'; ACC: sense5'-AGACAAACAGGGACCATT-3', antisense5'-AGGGACTGCCGAAACAT-3'; SCD: sense5'-CTGTGGAGTCACCGAACC-3', antisense5'-TAGCGTGGAACCCTTTT-3'; $\beta$-actin: sense5'-AAGGACCTCTACGCCAACACG-3', antisense5'-TTTGCGGTGGACGATGGAG-3'. All RT-PCR reactions were performed according to the previous report [49]. RT-PCR analysis was performed by the comparative CT method.

\subsection{Immunofluorescence}

The DCMECs were seeded on glass coverslips to 30-50\% confluency in six-well plates. They were then transiently transfected with pGCMV-IRES-EGFP-SOCS3 expression vector or siRNA targeted to SOCS3 and incubated for $24 \mathrm{~h}$, then cells were washed twice with PBS and fixed in $4 \%(w / v)$ ice-cold paraformaldehyde at $4{ }^{\circ} \mathrm{C}$ for $10 \mathrm{~min}$. Next, fixed cells were blocked in blocking buffer (in tris-buffered saline with $5 \%$ BSA and $0.1 \%$ TritonX-100) for $1 \mathrm{~h}$ at $37^{\circ} \mathrm{C}$, then incubated with either anti-SOCS3 primary antibody or anti-p-STAT5a primary antibody at 1:50 dilution for $1 \mathrm{~h}$ at $37^{\circ} \mathrm{C}$. After washing 
three times in $\mathrm{TBS} / \mathrm{T}$, specimens were incubated in the dark with FITC-conjugated anti-rabbit IgG at 1:200 dilution for $1 \mathrm{~h}$ at $37{ }^{\circ} \mathrm{C}$ and DAPI for $10 \mathrm{~min}$ at $37{ }^{\circ} \mathrm{C}$. Finally, after washing three times in TBS/T, the coverslips were mounted onto confocal microscopy with a Leica TCS-SP2 AOBS confocal microscope to detect SOCS3, p-STAT5a expression, respectively.

\subsection{Western Blot Analysis}

Western blot analysis was performed using standard techniques reported by LiMin Lu [48]. Total cell lysate containing about $30 \mu \mathrm{g}$ protein was separated on 10\% SDS-PAGE gel and transferred onto nitrocellulose membranes (Bio-RAD, Shanghai, China). Membranes were blocked in 5\% skim milk (in tris-buffered saline with 5\% skim milk and 0.1\% Tween-20). Membranes were probed with primary antibodies specific for the following antibodies: SOCS3(H-103; Santa Cruz Biotechnology Inc., Santa Cruz, CA, USA), STAT5a, p-STAT5a, mTOR, p-mTOR (Cell Signaling Technology, Beverly, MA, USA), SREBP-1c (the mature form, 68kD) (Santa Cruz), $\beta$-casein (Abbiotec, USA) and $\beta$-actin (Santa Cruz), followed by a second incubation with secondary antibodies [1:1000] conjugated to HRP (ZSGB-BIO, Beijing, China). The chemiluminescence detection of HRP-conjugated secondary antibodies was performed using Super ECL plus (ApplyGEN, Beijing, China).

\subsection{Construction of SOCS3 Eukaryotic Expression Plasmid and Transfection}

Total RNA from cultured DCMECs was extracted using Trizol reagent (Invitrogen), The cDNA was synthesized and the PCR product was inserted into pMD18-T plasmids (TaKaRa Company), followed by identifying with restriction enzyme EcoR I and Sal I (TaKaRa Company) and DNA sequencing. The SOCS3 gene was cloned into pGCMV-IRES-EGFP vector (GenePharma Co. Ltd, Shanghai, China). Therefore combinant plasmids were obtained and identified by digestion with EcoR I and Sal I. The following primers were designed with particular restriction enzyme sites to clone the complete coding region of SOCS3. Forward primer 5'-CGGAATTCATGGTCACCCACAGCAAGTT-3' $(E c o \mathrm{R}$ I) and reverse primer 5'-ACGCGTCGACCTAAAGCGGGGCATCGTACT-3' (Sal I). The optimized amplification conditions were annealing at $58.9^{\circ} \mathrm{C}$ and extension at $72{ }^{\circ} \mathrm{C}$ for 35 cycles.

Transient transfections was according to the previous report of $\mathrm{Lu}$ [48]. Briefly, DCMECs were transfected with pGCMV-IRES-EGFP-SOCS3 or empty vectors which was added to balance the total amounts of transfected DNA samples using Lipofectamine TM 2000 (LF2000, Invitrogen,) according to the manufacturer's protocol. Nontransfected cells were conducted as controls in the same way of transfection. Cells were cultivated for $48 \mathrm{~h}$. The cells were then collected for further experiments.

\subsection{Small Interfering RNA Transfection}

Small interfering RNA (transfection SOCS3 or STAT5a siRNA, GenePharma Co., Ltd.) and negative control RNA-oligonucleotides (GenePharma Co., Ltd.). DCMECs were transfected with the SOCS3 or STAT5a siRNA and negative control using Lipofectamine TM 2000 (LF2000, Invitrogen) according to the manufacturer's protocol. For silencing of SOCS3 or STAT5 gene expression, transfected cells were cultivated for $24 \mathrm{~h}$. The cells were then collected for further experiments. 


\subsection{Statistical Analysis}

Results were reported as mean \pm SE. All the data were obtained from at least three independent experiments. Data statistics and individual differences among groups were analyzed using $t$-test by Sigma Plot 9.0 software, and differences were considered statistically significant if $p<0.05$ or $p<0.01$.

\section{Conclusions}

SOCS3 inhibited $\beta$-casein gene expression and cell proliferation as an negative feedback-loop regulator of JAK2/STAT5 signaling pathway in DCMECs. Met can up-regulate milk protein synthesis by inhibition of expression of SOCS3. STAT5 is required for SOCS3 activation in DCMECs. We also noted a change in mTOR and SREBP-1c expression, due to activation and inhibition of SOCS3.

\section{Acknowledgments}

This study was financially supported by Major State Basic Research Development Program of China (973 Program, No. 2011CB100804), 863 Project of Ministry of Science and Technology of China (No. 2013AA102504-03).

\section{Conflicts of Interest}

There are no conflicts of interest.

\section{References}

1. Starr, R.; Willson, T.A.; Viney, E.M.; Murray, L.; Rayner, J.R.; Jenkins, B.J.; Gonda, T.J.; Alexander, W.S.; Metcalf, D.; Nicola, N.A. A family of cytokine-inducible inhibitors of signalling. Nature 1997, 387, 917-921.

2. Naka, T.; Narazaki, M.; Hirata, M.; Matsumoto, T.; Minamoto, S.; Aono, A.; Nishimoto, N.; Kajita, T.; Taga, T.; Yoshizaki, K. STAT-induced STAT inhibitor. Nature 1997, 387, 924-929.

3. Endo, T.A.; Masuhara, M.; Yokouchi, M.; Suzuki, R.; Sakamoto, H.; Mitsui, K.; Matsumoto, A.; Tanimura, S.; Ohtsubo, M.; Misawa, H. A new protein containing an SH2 domain that inhibits JAK kinases. Nature 1997, 387, 921-924.

4. Sachithanandan, N.; Fam, B.C.; Fynch, S.; Dzamko, N.; Watt, M.J.; Wormald, S.; Honeyman, J.; Galic, S.; Proietto, J.; Andrikopoulos, S. Liver-pecific suppressor of cytokine signaling-3 deletion in mice enhances hepatic insulin sensitivity and lipogenesis resulting in fatty liver and obesity 1. Hepatology 2010, 52, 1632-1642.

5. Trengove, M.C.; Ward, A.C. SOCS proteins in development and disease. Am. J. Clin. Exp. Immunol. 2013, 2, 1-29.

6. Yoshimura, A.; Naka, T.; Kubo, M. SOCS proteins, cytokine signalling and immune regulation. Nat. Rev. Immunol. 2007, 7, 454-465.

7. Tonko-Geymayer, S.; Goupille, O.; Tonko, M.; Soratroi, C.; Yoshimura, A.; Streuli, C.; Ziemiecki, A.; Kofler, R.; Doppler, W. Regulation and function of the cytokine-inducible SH-2 domain proteins, CIS and SOCS3, in mammary epithelial cells. Mol. Endocrinol. 2002, 16, 1680-1695. 
8. Hadsell, D.L.; Parlow, A.F.; Torres, D.; George, J.; Olea, W. Enhancement of maternal lactation performance during prolonged lactation in the mouse by mouse GH and long-R3-IGF-I is linked to changes in mammary signaling and gene expression. J. Endocrinol. 2008, 198, 61-70.

9. Barclay, J.L.; Anderson, S.T.; Waters, M.J.; Curlewis, J.D. SOCS3 as a tumor suppressor in breast cancer cells, and its regulation by PRL. Int. J. Cancer 2009, 124, 1756-1766.

10. Anderson, S.T.; Isa, N.N.; Barclay, J.L.; Waters, M.J.; Curlewis, J.D. Maximal expression of suppressors of cytokine signaling in the rat ovary occurs in late pregnancy. Reproduction 2009, 138, 537-544.

11. Robinson, G.W.; Pacher-Zavisin, M.; Zhu, B.M.; Yoshimura, A.; Hennighausen, L. Socs3 modulates the activity of the transcription factor Stat3 in mammary tissue and controls alveolar homeostasis. Dev. Dyn. 2007, 236, 654-661.

12. Dif, F.; Saunier, E.; Demeneix, B.; Kelly, P.A.; Edery, M. Cytokine-inducible SH2-containing protein suppresses PRL signaling by binding the PRL receptor. Endocrinology 2001, 142, 5286-5293.

13. Paukku, K.; Silvennoinen, O. STATs as critical mediators of signal transduction and transcription: Lessons learned from STAT5. Cytokine Growth Factor Rev. 2004, 15, 435-455.

14. Barash, I. Stat5 in the mammary gland: controlling normal development and cancer. J. Cell. Physiol. 2006, 209, 305-313.

15. Dong, B.; Zhao, F.Q. Involvement of the ubiquitous Oct-1 transcription factor in hormonal induction of beta-casein gene expression. Biochem. J. 2007, 401, 57-64.

16. Khatib, H.; Monson, R.; Schutzkus, V.; Kohl, D.; Rosa, G.; Rutledge, J. Mutations in the STAT5A gene are associated with embryonic survival and milk composition in cattle. J. Dairy Sci. 2008, 91, 784-793.

17. He, F.; Sun, D.X; Yu, Y. SNPs detection of STAT5A gene and association with milk production traits in Holstein cattle. Chin. J. Anim. Vet. Sci. 2007, 38, 326-331.

18. Bionaz, M.; Loor, J.J. Gene networks driving bovine mammary protein synthesis during the lactation cycle. Bioinforma. Biol. Insights 2011, 5, 83-98.

19. Bakan, I.; Laplante, M. Connecting mTORC1 signaling to SREBP-1 activation. Curr. Opin. Lipidol. 2012, 23, 226-234.

20. Yamamoto, T.; Watanabe, K.; Inoue, N.; Nakagawa, Y.; Ishigaki, N.; Matsuzaka, T.; Takeuchi, Y.; Kobayashi, K.; Yatoh, S.; Takahashi, A. Protein kinase Cbeta mediates hepatic induction of sterol-regulatory element binding protein-1c by insulin. J. Lipid Res. 2010, 51, 1859-1870.

21. Ueki, K.; Kadowaki, T.; Kahn, C.R. Role of suppressors of cytokine signaling SOCS-1 and SOCS-3 in hepatic steatosis and the metabolic syndrome. Hepatol. Res. 2005, 33, 185-192.

22. Orellana, R.A.; Jeyapalan, A.; Escobar, J.; Frank, J.W.; Nguyen, H.V.; Suryawan, A.; Davis, T.A. Amino acids augment muscle protein synthesis in neonatal pigs during acute endotoxemia by stimulating mTOR-dependent translation initiation. Am. J. Physiol.-Endocrinol. Metab. 2007, 293, E1416-E1425.

23. Correa-Fiz, F.; Reyes-Palomares, A.; Fajardo, I.; Melgarejo, E.; Gutierrez, A.; GarcIa-Ranea, J.A.; Medina, M.A.; Sanchez-Jimenez, F. Regulatory cross-talk of mouse liver polyamine and methionine metabolic pathways: A systemic approach to its physiopathological consequences. Amino Acids 2011, 42, 577-595. 
24. Honda, M.; Takehana, K.; Sakai, A.; Tagata, Y.; Shirasaki, T.; Nishitani, S.; Muramatsu, T.; Yamashita, T.; Nakamoto, Y.; Mizukoshi, E. Malnutrition impairs interferon signaling through mTOR and FoxO pathways in patients with chronic hepatitis C. Gastroenterology 2011, 141, 128-140.

25. Lu, L.M.; Gao, X.J.; Li, Q.Z.; Huang, J.G.; Liu, R.; Li, H.M. Comparative phosphoproteomics analysis of the effects of L-methionine on dairy cow mammary epithelial cells. Can. J. Anim. Sci. 2012, 92, 433-442.

26. Peltola, K.J.; Paukku, K.; Aho, T.L.; Ruuska, M.; Silvennoinen, O.; Koskinen, P.J. Pim-1 kinase inhibits STAT5-dependent transcription via its interactions with SOCS1 and SOCS3. Blood 2004, 103, 3744-3750.

27. Helman, D.; Sandowski, Y.; Cohen, Y.; Matsumoto, A.; Yoshimura, A.; Merchav, S.; Gertler, A. Cytokine-inducible $\mathrm{SH} 2$ protein (CIS3) and JAK2 binding protein (JAB) abolish prolactin receptor-mediated STAT5 signaling. FEBS Lett. 1998, 441, 287-291.

28. Tam, S.P.; Lau, P.; Djiane, J.; Hilton, D.J.; Waters, M.J. Tissue-specific induction of SOCS gene expression by PRL. Endocrinology 2001, 142, 5015-5026.

29. White, G.E.; Cotterill, A.; Addley, M.R.; Soilleux, E.J.; Greaves, D.R. Suppressor of cytokine signalling protein SOCS3 expression is increased at sites of acute and chronic inflammation. J. Mol. Histol. 2011, 42, 137-151.

30. Jiang, S.Z.; Ren, Z.R.; Xie, F.; Yan, J.B.; Huang, S.Z.; Zeng, Y.T. Bovine prolactin elevates hTF expression directed by a tissue-specific goat $\beta$-casein promoter through prolactin receptor-mediated STAT5a activation. Biotechnol. Lett. 2012, 34, 1991-1999.

31. He, X.; Chu, M.X.; Qiao, L.; He, J.N.; Wang, P.Q.; Feng, T.; Di, R.; Cao, G.L.; Fang, L.; An, Y.F. Polymorphisms of STAT5A gene and their association with milk production traits in Holstein cows. Mol. Biol. Rep. 2012, 39, 2901-2907.

32. Chen, C.-C.; Boxer, R.B.; Stairs, D.B.; Portocarrero, C.P.; Horton, R.H.; Alvarez, J.V.; Birnbaum, M.J.; Chodosh, L.A. Akt is required for Stat5 activation and mammary differentiation. Breast Cancer Res. 2010, 12, R72.

33. Cui, Y.; Riedlinger, G.; Miyoshi, K.; Tang, W.; Li, C.; Deng, C.-X.; Robinson, G.W.; Hennighausen, L. Inactivation of Stat5 in mouse mammary epithelium during pregnancy reveals distinct functions in cell proliferation, survival, and differentiation. Mol. Cell. Biol. 2004, 24, 8037-8047.

34. Lee, K.-H.; Moon, K.-J.; Kim, H.S.; Yoo, B.C.; Park, S.; Lee, H.; Kwon, S.; Lee, E.S.; Yoon, S. Increased cytoplasmic levels of CIS, SOCS1, SOCS2, or SOCS3 are required for nuclear translocation. FEBS Lett. 2008, 582, 2319-2324.

35. Ehrentraut, S.; Nagel, S.; Scherr, M.E.; Schneider, B.; Quentmeier, H.; Geffers, R.; Kaufmann, M.; Meyer, C.; Prochorec-Sobieszek, M.; Ketterling, R.P. t (8; 9)(p22; p24)/PCM1-JAK2 activates SOCS2 and SOCS3 via STAT5. PLoS One 2013, 8, e53767.

36. Le Provost, F.; Miyoshi, K.; Vilotte, J.-L.; Bierie, B.; Robinson, G.W.; Hennighausen, L. SOCS3 promotes apoptosis of mammary differentiated cells. Biochem. Biophys. Res. Commun. 2005, 338, 1696-1701.

37. Chueh, F.-Y.; Leong, K.-F.; Cronk, R.J.; Venkitachalam, S.; Pabich, S.; Yu, C.-L. Nuclear localization of pyruvate dehydrogenase complex-E2 (PDC-E2), a mitochondrial enzyme, and its role in signal transducer and activator of transcription 5 (STAT5)-dependent gene transcription. Cell Signal. 2011, 23, 1170-1178. 
38. Toerien, C.A.; Trout, D.R.; Cant, J.P. Nutritional stimulation of milk protein yield of cows is associated with changes in phosphorylation of mammary eukaryotic initiation factor 2 and ribosomal s6 kinase 1. J. Nutr. 2010, 140, 285-292.

39. Galbaugh, T.; Cerrito, M.G.; Jose, C.C.; Cutler, M.L. EGF-induced activation of Akt results in mTOR-dependent p70S6 kinase phosphorylation and inhibition of $\mathrm{HC} 11$ cell lactogenic differentiation. BMC Cell Biol. 2006, 7, 34.

40. Stahl, A.; Joyal, J.-S.; Chen, J.; Sapieha, P.; Juan, A.M.; Hatton, C.J.; Pei, D.T.; Hurst, C.G.; Seaward, M.R.; Krah, N.M. SOCS3 is an endogenous inhibitor of pathologic angiogenesis. Blood 2012, 120, 2925-2929.

41. Boutinaud, M.; Guinard-Flament, J.; Jammes, H. The number and activity of mammary epithelial cells, determining factors for milk production. Reprod. Nutr. Dev. 2004, 44, 499-508.

42. He, B.; You, L.; Uematsu, K.; Zang, K.; Xu, Z.; Lee, A.Y.; Costello, J.F.; McCormick, F.; Jablons, D.M. SOCS-3 is frequently silenced by hypermethylation and suppresses cell growth in human lung cancer. Proc. Natl. Acad. Sci. USA 2003, 100, 14133-14138.

43. Elam, M.B.; Yellaturu, C.; Howell, G.E.; Deng, X.; Cowan, G.S.; Kumar, P.; Park, E.A.; Hiler, M.L.; Wilcox, H.G.; Hughes, T.A. Dysregulation of sterol regulatory element binding protein-1c in livers of morbidly obese women is associated with altered suppressor of cytokine signaling-3 and signal transducer and activator of transcription-1 signaling. Metabolism 2010, 59, 587-598.

44. Ueki, K.; Kondo, T.; Tseng, Y.-H.; Kahn, C.R. Central role of suppressors of cytokine signaling proteins in hepatic steatosis, insulin resistance, and the metabolic syndrome in the mouse. Proc. Natl. Acad. Sci. USA 2004, 101, 10422-10427.

45. Ma, L.; Corl, B.A. Transcriptional regulation of lipid synthesis in bovine mammary epithelial cells by sterol regulatory element binding protein-1. J. Dairy Sci. 2012, 95, 3743-3755.

46. Han, J.M.; Jeong, S.J.; Park, M.C.; Kim, G.; Kwon, N.H.; Kim, H.K.; Ha, S.H.; Ryu, S.H.; Kim, S. Leucyl-tRNA synthetase is an intracellular leucine sensor for the mTORC1-signaling pathway. Cell 2012, 149, 410-424.

47. Prizant, R.L.; Barash, I. Negative effects of the amino acids Lys, His, and Thr on S6K1 phosphorylation in mammary epithelial cells. J. Cell. Biochem. 2008, 105, 1038-1047.

48. Lu, L.M.; Li, Q.Z.; Huang, J.G.; Gao, X.J. Proteomic and functional analyses reveal MAPK1 regulates milk protein synthesis. Molecules 2013, 18, 263-275.

49. Huang, J.G.; Gao, X.J.; Li, Q.Z.; Lu, L.M.; Liu, R.; Luo, C.C.; Wang, J.L.; Qiao, B.; Jin, X. Proteomic analysis of the nuclear phosphorylated proteins in dairy cow mammary epithelial cells treated with estrogen. In Vitro Cell. Dev. Biol.-Anim. 2012, 48, 449-457.

Sample Availability: Samples of the dairy cow mammary epithelial cells (DCMECs) are available from the authors.

(C) 2013 by the authors; licensee MDPI, Basel, Switzerland. This article is an open access article distributed under the terms and conditions of the Creative Commons Attribution license (http://creativecommons.org/licenses/by/3.0/). 